\title{
Giant magnetocaloric effects by tailoring the phase transitions
}

\author{
N. T. Trung, L. Zhang, L. Caron, K. H. J. Buschow, and E. Brück ${ }^{\text {a) }}$ \\ Fundamental Aspects of Materials and Energy, Faculty of Applied Sciences, TU Delft, Mekelweg 15, \\ 2629 JB Delft, The Netherlands
}

(Received 18 January 2010; accepted 28 March 2010; published online 27 April 2010)

\begin{abstract}
The MnCoGe alloy can crystallize in either the hexagonal $\mathrm{Ni}_{2} \mathrm{In}$ - or the orthorhombic TiNiSi-type of structure. In both phases MnCoGe behaves like a typical ferromagnet with a second-order magnetic phase transition. For $\mathrm{MnCoGeB}_{\mathrm{x}}$ with $\mathrm{B}$ on interstitial positions, we discover a giant magnetocaloric effect associated with a single first-order magnetostructural phase transition, which can be achieved by tuning the magnetic and structural transitions to coincide. The results obtained on the MnCoGe-type alloys may be extensible to other types of magnetic materials undergoing a first-order structural transformation and can open up some possibilities for searching magnetic refrigerants for room-temperature applications. (C) 2010 American Institute of Physics.
\end{abstract}

[doi:10.1063/1.3399773]

Magnetic refrigeration based on the magnetocaloric effect $(\mathrm{MCE})$ is a possible alternative to the current vaporcompression technology. ${ }^{1}$ Nowadays, most studies on magnetic refrigerants are focused on materials undergoing a firstorder magnetic phase transition because of their potential applications at room temperature $\left(T_{\text {room }}\right) .^{2-6}$ However, for different reasons none of these materials have yet been employed in a commercial refrigerator. Obviously, there is a need for yet further improved materials.

Studies made on MnCoGe confirmed a diffusionless, martensitic transformation from the low-temperature orthorhombic (orth.) TiNiSi-type structure (space group Pnma) to the high-temperature hexagonal (hex.) $\mathrm{Ni}_{2}$ In-type structure (space group $\mathrm{Pb}_{3} / \mathrm{mmc}$ ). ${ }^{7,8}$ On heating this structural transformation occurs at $\sim 650 \mathrm{~K},{ }^{8}$ and the transition temperature $\left(T_{\mathrm{t}}\right)$ can be changed by either changing the composition ${ }^{8-12}$ or applying an external pressure. ${ }^{13-15}$ A metastable lowtemperature state of the hex. phase can be formed at ambient conditions by quenching the material from high temperatures. ${ }^{13,16}$ On the other hand, a strong correlation between the magnetic and the structural behavior of $\mathrm{MnCoGe}$ was observed. ${ }^{16}$ In both the orth. and the hex. structure, MnCoGe behaves like a ferromagnet with a second-order magnetic phase transition but the magnetic saturation moment $\left(M_{\mathrm{S}}\right)$ and the magnetic ordering temperature $\left(T_{\mathrm{c}}\right)$ are different for these two phases. In the hex. structure, the values of $M_{\mathrm{S}}$ and $T_{\mathrm{c}}$ of $\mathrm{MnCoGe}$ are $2.76 \mu_{\mathrm{B}}$ and $275 \mathrm{~K}$, respectively. ${ }^{9}$ In the orth. form, $\mathrm{MnCoGe}$ exhibits a higher $M_{\mathrm{S}}$ and also a higher $T_{\mathrm{c}}$, namely $4.13 \mu_{\mathrm{B}}$ and $345 \mathrm{~K}$, respectively. ${ }^{17}$ Note that the $T_{\mathrm{c}}$ and the $T_{\mathrm{t}}$ of the orth. MnCoGe are a few hundred degrees separated. Therefore, a magnetostructural coupling cannot take place in this case.

The magnetocaloric materials based on MnCoGe described in this paper is especially suited for operation between 275 and $345 \mathrm{~K}$. Special attention is paid to the mechanism of manipulating a first-order magnetostructural transition (FOMST) in the $\mathrm{MnCoGeB}_{\mathrm{x}}$ compounds, leading to giant MCEs and involving a single paramagnetic (PM) $\mathrm{Ni}_{2}$ In-type phase transforming reversibly into a ferromagnetic (FM) composite consisting of both the $\mathrm{Ni}_{2}$ In-type and

${ }^{a)}$ Electronic mail: e.h.brueck@tudelft.nl.
TiNiSi-type phases. An essential ingredient for the studied materials is the discovery that it is possible to shift the $T_{\mathrm{t}}$ value between the orth. and the hex. phase from about $650 \mathrm{~K}$ down to $T_{\text {room }}$ using a few percent of interstitial atoms as additives.

Ingots of polycrystalline MnCoGe-based alloys were prepared by arc-melting appropriate amounts of the highpurity constituent elements under Ar atmosphere in a watercooled copper crucible. The samples were annealed at $850{ }^{\circ} \mathrm{C}$ for $120 \mathrm{~h}$ and then quenched into water at $T_{\text {room}}$. Temperature dependence of powder X-ray diffraction (XRD) was measured in an X'pert Pro PANalytical diffractometer with $\mathrm{Cu} K \alpha$ radiation. The lattice parameters and the volume fraction (vol \%) of the orth. and the hex. phase were calculated by Rietveld refinement using the FULLPROF package. ${ }^{18}$ Magnetic measurements were performed in a commercial superconducting quantum interference device magnetometer (Quantum Design MPMS 5XL).

Shown in Fig. 1(a) are the temperature dependences of the magnetization ( $M-T$ curves) of the $\mathrm{MnCoGeB}_{\mathrm{x}}$ $(x=0.02,0.03,0.05)$ alloys. For the samples with $x=0.02$ and 0.03 , the sharp magnetic transitions represent an abrupt change of magnetization from the low-temperature FM to the high-temperature PM state. Also, thermal hysteresis $\left(\Delta T_{\text {hys }}\right)$ can be observed between the heating and cooling processes, indicating the first-order nature of these transitions. As has been shown recently, the magnetic entropy change $\left(\Delta S_{\mathrm{m}}\right)$ of materials with hysteretic first-order transitions can reliably be calculated via the Maxwell relation. ${ }^{1,19}$ For $\Delta B$ $=0-5 \mathrm{~T}$, we obtain giant MCEs of $-47.3 \mathrm{~J} \mathrm{~kg}^{-1} \mathrm{~K}^{-1}$ and $-37.7 \mathrm{~J} \mathrm{~kg}^{-1} \mathrm{~K}^{-1}$ for $\mathrm{x}=0.02$ and $\mathrm{x}=0.03$, respectively. Also, the saturation moments of these samples $\left(M_{\mathrm{S}}\right.$ $\sim 3.85 \mu_{\mathrm{B}}$ ) are lower than that found in the un-doped MnCoGe. ${ }^{17}$ However, with further increase in the boron concentration, as for the sample with $\mathrm{x}=0.05$, the MCE magnitude drops to $-3.4 \mathrm{~J} \mathrm{~kg}^{-1} \mathrm{~K}^{-1}$ near $T_{\mathrm{c}}=263 \mathrm{~K}$ and the $M_{\mathrm{S}}$ value is reduced to about $2.78 \mu_{\mathrm{B}}$ [see Fig. 1(b) and Table I].

Figure 2 shows XRD patterns measured at 100, 250, and $400 \mathrm{~K}$ of the $\mathrm{x}=0.02$ sample. The structural change is evident from these patterns. In connection with the $M-T$ curve of this sample, this observation is in accordance with a FOMST, namely a coupling between the magnetic and struc- 

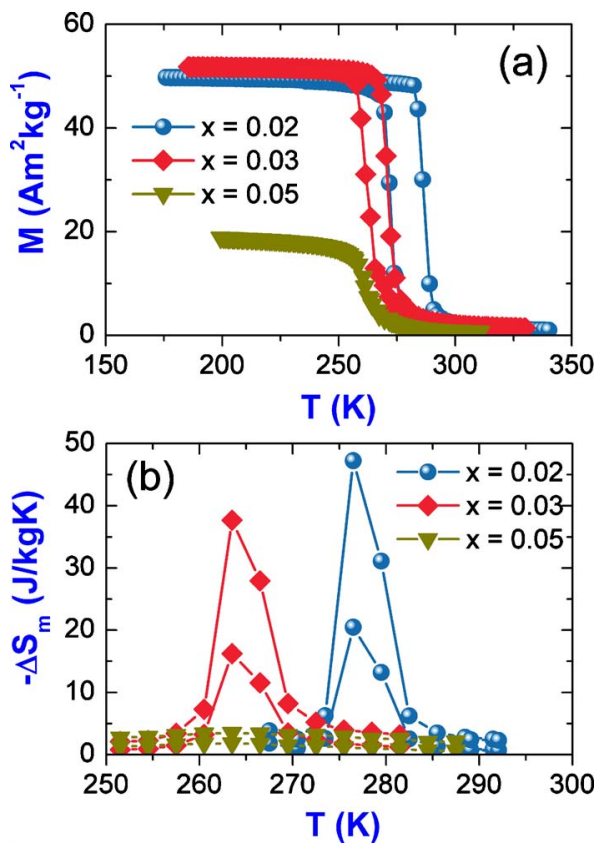

FIG. 1. (Color online) $M-T$ curves measured in magnetic field $\mathrm{B}=0.1 \mathrm{~T}$ of the $\mathrm{MnCoGeB}_{\mathrm{x}}(\mathrm{x}=0.02,0.03,0.05)$ samples (a) and their corresponding $\Delta S_{\mathrm{m}}$ as a function of temperature under the field change of $\Delta B=0-2 \mathrm{~T}$ (lower curves) and $\Delta B=0-5 \mathrm{~T}$ (upper curves) (b).

tural transitions. ${ }^{2-6}$ Shown in Fig. 3 is the temperature dependence of the orth. phase fraction as derived from the refinement of XRD patterns obtained in zero-field for the $\mathrm{x}=0.02$ sample. The sharp structural transition and the $\Delta T_{\text {hys }}$ value of $\sim 15 \mathrm{~K}$ are in agreement with the magnetization measurements shown in Fig. 1(a). Apparently, the addition of interstitial $\mathrm{B}$ atoms in the $\mathrm{MnCoGeB}_{\mathrm{x}}$ alloys has stabilized the hex. phase relative to the orth. phase so that the stability region of the former has become enlarged. It extends to much lower temperatures than that for the parent compound MnCoGe. ${ }^{7,8}$ This offers the possibility that for a properly chosen boron concentration the structural and magnetic phase transitions can be controlled to coincide, leading to a single FOMST with a large MCE. As can also be seen in Fig. 3 , the structural transition is not complete. Below $T_{c}$, a relatively small amount of the hex. phase still remains when cooling to temperatures below the phase transition. The hex. phase fraction calculated at $100 \mathrm{~K}$ is about $11.3 \mathrm{vol} \%$ for the

TABLE I. Values of $T_{\mathrm{c}}, \Delta T_{\text {hys }}$, maximal $\Delta S_{\mathrm{m}}$ under the field change $\Delta B$ $=0-5 \mathrm{~T}, M_{\mathrm{S}}$ measured at $5 \mathrm{~K}$, and the low-temperature phase fraction (vol \%) of the orth. and hex. structures obtained from the XRD measured at $100 \mathrm{~K}$ for some MnCoGe-type alloys.

\begin{tabular}{lcccccrr}
\hline \hline & & & & & \multicolumn{3}{c}{$\begin{array}{c}\text { Phase fraction } \\
\text { (vol \%) }\end{array}$} \\
\cline { 7 - 9 } Samples & $\begin{array}{c}T_{\mathrm{c}} \\
(\mathrm{K})\end{array}$ & $\begin{array}{c}\Delta T_{\text {hys }} \\
(\mathrm{K})\end{array}$ & $\begin{array}{c}-\Delta S_{\mathrm{m}, \max } \\
\left(\mathrm{J} \mathrm{kg}^{-1} \mathrm{~K}^{-1}\right)\end{array}$ & $\begin{array}{c}M_{\mathrm{S}} \\
\left(\mu_{\mathrm{B}} / \text { f.u. }\right)\end{array}$ & \multicolumn{1}{c}{ Orth. } & Hex. \\
\hline $\mathrm{MnCoGe}^{\mathrm{a}}$ & 345 & 0 & 5.6 & 4.13 & 100.0 & 0.0 \\
$\mathrm{MnCoGeB}_{0.01}$ & 304 & 9 & 14.6 & 3.86 & 93.3 & 6.7 \\
$\mathrm{MnCoGeB}_{0.02}$ & 287 & 14 & 47.3 & 3.86 & 88.7 & 11.3 \\
$\mathrm{MnCoGeB}_{0.03}$ & 275 & 9 & 37.7 & 3.85 & 83.8 & 16.2 \\
$\mathrm{MnCoGeB}_{0.05}$ & 260 & 0 & 3.4 & 2.78 & 0.0 & 100.0 \\
$\mathrm{Mn}_{0.98} \mathrm{CoGe}$ & 302 & 11 & 30.2 & 3.96 & 92.7 & 7.3 \\
$\mathrm{MnCoGeC}_{0.03}$ & 342 & 15 & 31.4 & 4.02 & 98.3 & 1.7 \\
\hline \hline
\end{tabular}

${ }^{\mathrm{a}}$ Reference 17.

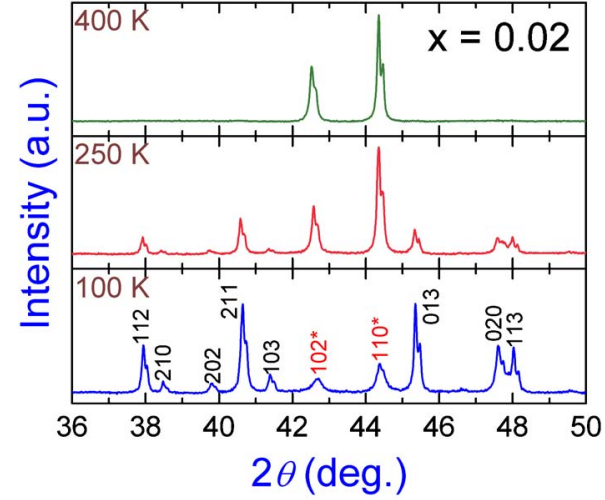

FIG. 2. (Color online) Some selected XRD patterns of the $\mathrm{x}=0.02$ sample measured at 100, 250, and $400 \mathrm{~K}$ in zero-field upon heating. At $100 \mathrm{~K}$, the pattern confirms the coexistence of the orth. phase ( $h k l$ Miller indices without *) and the hex. phase ( $h k l$ Miller indices with *).

$\mathrm{x}=0.02$ sample. We attribute this to boron concentration fluctuation where the structural phase transition in relatively boron rich regions does not occur. The presence of hex. phase below $T_{\mathrm{c}}$ can make this phase grow favorably when the sample is heated up to above $T_{\mathrm{c}}$. In other words, the coexistence of the orth. and hex. structures below $T_{\mathrm{c}}$ is favorable for the FOMST. On the other hand, from the crystallographic point of view, the TiNiSi-type unit-cell can be considered as an orth. distortion of the hex. $\mathrm{Ni}_{2}$ In-type unit-cell. The relationship between their lattice constants and unit-cell volumes are given by $a_{\text {orth }}=c_{\text {hex }}, b_{\text {orth }}=a_{\text {hex }}, c_{\text {orth }}=\sqrt{3} a_{\text {hex }}$, and $V_{\text {orth }}=2 V_{\text {hex }} \cdot{ }^{8-10}$ For $\mathrm{x}=0.02$, the structural transition is relatively broader than the magnetic transition [see Figs. 1(a) and 3]. Probably, the response of magnetic moments in the presence of magnetic field is faster than the structural distortion in zero-field when the material is going through the transition point.

The $x=0.05$ sample exhibits a single phase of the hex. structure down to temperatures below $T_{\mathrm{c}}$. In this case, the magnetostructural coupling and the giant $\mathrm{MCE}$ are no longer observed. Here the higher boron concentration has further extended the stability range of the hex. phase preventing the simultaneous occurrence of the structural and magnetic phase transitions. The concomitant loss of the first-order character of the transition leads to the disappearance of the giant MCE.

It was above mentioned that the FOMST plays an essential role in giving rise to the giant MCEs observed in the

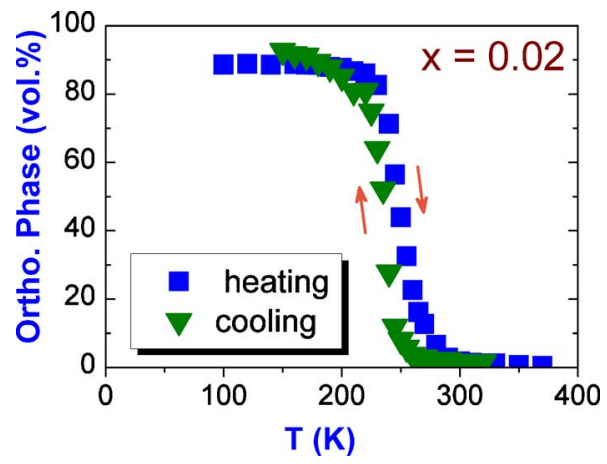

FIG. 3. (Color online) Temperature dependence of the phase fraction (vol \%) of the orth. structure as derived from XRD patterns measured with increasing and decreasing temperature for the $\mathrm{x}=0.02$ sample. 
$\mathrm{MnCoGeB}_{\mathrm{x}}$ alloys. The structural and magnetic results obtained on boron-doped $\mathrm{MnCoGe}$ are summarized as follow. ${ }^{20}$ For boron concentrations $\mathrm{x} \leq 0.05$ at. \%, accompanying by quenching, we can shift the martensitic transition temperature $T_{\mathrm{t}}$ from about $650 \mathrm{~K}$ to far below $T_{\mathrm{c}}$ of the two allotropic phases of MnCoGe. By choosing the concentration of boron atoms in the range $0.02-0.03$ at. \% we can achieve an intimate coupling between the magnetic and structural phasetransitions. Concerning practical application as refrigerants, an optimal distribution of the boron atoms in the lattice and the corresponding coexistence of the two allotropic phases (albeit in temperature-dependent proportions, see Fig. 3) are essential for good functioning of the presently discovered giant-MCE materials. Whereas, a martensitic-type transformation is usually characterized by a strong $\Delta T_{\text {hys }}{ }^{8,21}$ the coexistence of both allotropic forms avoids this thermal hysteresis. One can see that the structural transition shown in Fig. 3 is quasi-reversible. The small $\Delta T_{\text {hys }}$ of the MnCoGe-type alloys shown Table I is most likely a signature of the firstorder character of the magnetostructural transition. We note that the FOMST and giant MCE can also be achieved on the introduction of $\mathrm{C}$ or Mn vacancies in $\mathrm{MnCoGe}$ (see Table I).

In conclusion, we have discovered giant MCE near $T_{\text {room }}$ in the magnetocaloric materials based on MnCoGe. By introducing interstitial boron atoms, the structural and magnetic transitions in $\mathrm{MnCoGe}$ can be controlled to coincide, leading to a single FOMST from the combined two-phase FM region, in which the TiNiSi- and $\mathrm{Ni}_{2}$ In-type structures coexist, to an almost single-phase $\mathrm{PM} \mathrm{Ni}_{2}$ In-type structure. The results obtained on the MnCoGe-based compounds may be extensible to other types of magnetic materials undergoing separate structural and magnetic transitions ${ }^{7}$ and can open up some possibilities for searching magnetic refrigerants for room-temperature applications.

Special thanks are due to M. P. Steenvoorden for his assistance on XRD experiments. The authors are grateful to H. Schlatter and A. J. Riemersma for their support in sample preparation. We thank A. J. E. Lefering and R. Blondé for critical reading of the manuscript. This work was financially supported by the Dutch Technology Foundation (STW) and the Foundation for Fundamental Research on Matter (FOM), which is financially supported by the Netherlands Organisation for Scientific Research (NWO).

${ }^{1}$ E. Brück, J. Phys. D 38, R381 (2005).

${ }^{2}$ V. K. Pecharsky and K. A. Gschneidner, Phys. Rev. Lett. 78, 4494 (1997).

${ }^{3}$ O. Tegus, E. Brück, K. H. J. Buschow, and F. R. de Boer, Nature (London) 415, 150 (2002).

${ }^{4}$ H. Wada and Y. Tanabe, Appl. Phys. Lett. 79, 3302 (2001)

${ }^{5}$ F. X. Hu, B. G. Shen, J. R. Sun, Z. H. Cheng, G. H. Rao, and X. X. Zhang, Appl. Phys. Lett. 78, 3675 (2001).

${ }^{6}$ T. Krenke, E. Duman, M. Acet, E. F. Wassermann, X. Moya, L. Mañosa, and A. Planes, Nature Mater. 4, 450 (2005).

${ }^{7}$ O. Beckman and L. Lundgren, in Handbook of Magnetic Materials, edited by K. H. J. Buschow (Elsevier, New York, 1991), Vol. 6, Chap. 3.

${ }^{8}$ T. Kanomata, H. Ishigaki, T. Suzuki, H. Yoshida, S. Abe, and T. Kaneko, J. Magn. Magn. Mater. 140-144, 131 (1995).

${ }^{9}$ J. T. Wang, D. S. Wang, C. Chen, O. Nashima, T. Kanomata, H. Mizuseki, and Y. Kawazoe, Appl. Phys. Lett. 89, 262504 (2006).

${ }^{10}$ K. Koyama, M. Sakai, T. Kanomata, and K. Watanabe, Jpn. J. Appl. Phys., Part 1 43, 8036 (2004).

${ }^{11}$ V. Johnson, Inorg. Chem. 14, 1117 (1975).

${ }^{12}$ S. Niziol, A. Weselucha, W. Bazela, and A. Szytula, Solid State Commun. 39, 1081 (1981).

${ }^{13}$ S. Niziol, A. Zieba, R. Zach, M. Baj, and L. Dmowski, J. Magn. Magn. Mater. 38, 205 (1983).

${ }^{14}$ S. Anzai and K. Ozawa, Phys. Rev. B 18, 2173 (1978).

${ }^{15}$ S. Niziol, R. Zach, J. P. Senateur, and J. Beille, J. Magn. Magn. Mater. 79, 333 (1989).

${ }^{16}$ S. Kaprzyk and S. Niziol, J. Magn. Magn. Mater. 87, 267 (1990).

${ }^{17}$ S. Lin, O. Tegus, E. Brück, W. Dagula, T. J. Gortenmulder, and K. H. J. Buschow, IEEE Trans. Magn. 42, 3776 (2006).

${ }^{18}$ J. Rodríguez-Carvajal, Physica B 192, 55 (1993).

${ }^{19}$ L. Caron, Z. Q. Ou, T. T. Nguyen, D. T. Cam Thanh, O. Tegus, and E. Brück, J. Magn. Magn. Mater. 321, 3559 (2009).

${ }^{20}$ See supplementary material at http://dx.doi.org/10.1063/1.3399773 for phase diagram and magnetic response of the $\mathrm{MnCoGeB}_{x}$.

${ }^{21}$ B. Hernando, J. L. Sánchez Llamazares, V. M. Prida, D. Baldomir, D. Serantes, M. Ilyn, and J. González, Appl. Phys. Lett. 94, 222502 (2009). 